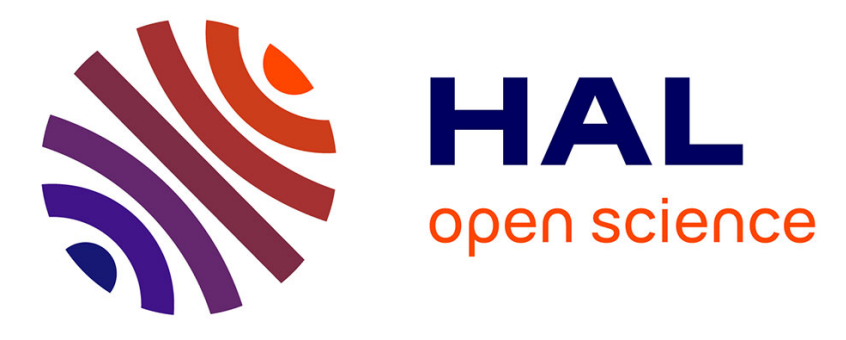

\title{
Aircraft trajectory management and control with spatial reference
}

\author{
Hakim Bouadi, Alexandre Carlos Brandao-Ramos, Thierry Miquel, Felix
}

Mora-Camino

\section{- To cite this version:}

Hakim Bouadi, Alexandre Carlos Brandao-Ramos, Thierry Miquel, Felix Mora-Camino. Aircraft trajectory management and control with spatial reference. ICCA 2013, 10th IEEE International Conference on Control and Automation, Jun 2013, Hangzhou, China. pp 1592-1597; ISBN : 978-14673-4707-5, 10.1109/ICCA.2013.6564909 . hal-00945176

\section{HAL Id: hal-00945176 \\ https://hal-enac.archives-ouvertes.fr/hal-00945176}

Submitted on 2 Jun 2014

HAL is a multi-disciplinary open access archive for the deposit and dissemination of scientific research documents, whether they are published or not. The documents may come from teaching and research institutions in France or abroad, or from public or private research centers.
L'archive ouverte pluridisciplinaire HAL, est destinée au dépôt et à la diffusion de documents scientifiques de niveau recherche, publiés ou non, émanant des établissements d'enseignement et de recherche français ou étrangers, des laboratoires publics ou privés. 


\title{
Aircraft Trajectory Management and Control with Spatial Reference
}

\author{
H. Bouadi, A.C. Brandão Ramos, T. Miquel and F. Mora-Camino
}

\begin{abstract}
The purpose of this communication is to investigate the interest of using a spatial reference for performing first aircraft trajectory generation and then trajectory tracking while overfly or arrival time constraints are imposed. The adoption of a space reference leads us to rewrite the aircraft flight dynamics. Then an aircraft trajectory optimization problem, including time constraints, is formulated and discussed. Then a new nonlinear control structure for trajectory tracking based on spatial reference is developed and simulation results are displayed.
\end{abstract}

Index Terms - Trajectory optimization, trajectory tracking, guidance dynamics, nonlinear control, space reference.

\section{INTRODUCTION}

$\mathrm{W}$ orld air transportation traffic has known a sustained increase over the last decades leading to airspace near saturation in large areas of developed and emerging countries. For example, today up to 27,000 flights cross European airspace every day while the number of passengers is expected to double by 2020 . Then safety and environmental considerations urge today for the development of new guidance systems with improved accuracy for spatial and temporal trajectory tracking. Available infrastructure of current ATM (Air Traffic Management) will no longer be able to stand this growing demand unless breakthrough improvements are made. In the future air traffic management environment defined by SESAR (Single European Sky ATM Research) and NextGen (Next Generation Air Transportation System) projects, two main objectives are targeted, strategic data link services for sharing of information and negotiation of planning constraints between ATC (Air Traffic Control) and

H. Bouadi is a PhD student at MAIAA Laboratory, Automation Research Group at ENAC, Toulouse, France, hakim.bouadi@enac.fr.

A.C. Brandão Ramos is an associate professor at UNIFEI, Mathematics and Computer Science Institute, Itajuba, Brazil, Tel.+553536291428, ramos@unifei.edu.br

T. Miquel and F. Mora-Camino are professor at MAIAA Laboratory, Automation Research Group at ENAC, Toulouse, France, Tel.+33562174358, fax. +33562174403, thierry.miquel@enac.fr, felix.mora@enac.fr. the aircraft in order to ensure planning consistency and the use of the 4D aircraft trajectory information in the flight management system for ATC operations [1], [2]. This means that in addition to following the trajectory cleared by ATC, aircraft will progress in four dimensions, sharing accurate airborne predictions with the ground systems, and being able to meet time constraints at specific waypoints with high precision when the traffic density requires it. This will allow better separation and sequencing of traffic flows while green climb/descent trajectories will be feasible in terminal areas.

In this communication we introduce a spatial reference to ease the way of these necessary development in air traffic control and management by providing new capabilities for aircraft by coping more efficiently with overfly and arrival time constraints. The trajectory optimization problem corresponding to reference trajectory generation at the level of flight management (FMS-Flight Management System), as well as $2 \mathrm{D}+\mathrm{T}$ trajectory tracking at the level of flight guidance (AGS-Auto Guidance System) are considered.

\section{Kinematics AlONG A Horizontal Track}

Let the speed of an aircraft be given first in the Earth frame $(E)$ and then in the body frame $(B)$ :

$$
\vec{V}=\left[\begin{array}{c}
V \cos \gamma \cos \psi \\
V \cos \gamma \sin \psi \\
-V \sin \gamma
\end{array}\right]_{E}=\left[\begin{array}{c}
V \cos \alpha \cos \beta \\
V \sin \beta \\
V \sin \alpha \cos \beta
\end{array}\right]_{B}
$$

where $V=\|\vec{V}\|, \gamma$ is the flight path angle and $\psi$ is the heading angle, $\alpha$ is the angle of attack and $\beta$ is the side slip angle [3].

Let $M(\theta, \phi, \psi)$ be the rotation matrix leading from the body frame to the local Earth frame where $\theta$ is the pitch angle and $\phi$ is the bank angle. This matrix is given by:

$$
M(\theta, \phi, \psi)=\left[\begin{array}{ccc}
c_{\theta} c_{\psi} & s_{\phi} s_{\theta} c_{\psi}-c_{\phi} s_{\psi} & c_{\phi} s_{\theta} c_{\psi}+s_{\phi} s_{\psi} \\
c_{\theta} s_{\psi} & s_{\phi} s_{\theta} s_{\psi}+c_{\phi} c_{\psi} & c_{\phi} s_{\theta} s_{\psi}-s_{\phi} s_{\psi} \\
-s_{\theta} & s_{\phi} s_{\theta} s_{\psi}+c_{\phi} c_{\psi} & c_{\phi} c_{\theta}
\end{array}\right]
$$


where $c_{\theta}=\cos \theta, s_{\phi}=\sin \phi$, and so on. Then we have:

$$
\left[\begin{array}{c}
V \cos \gamma \cos \mu \\
V \cos \gamma \sin \mu \\
-V \sin \gamma
\end{array}\right]=M(\theta, \phi, \psi)\left[\begin{array}{c}
V \cos \alpha \cos \beta \\
V \sin \beta \\
V \sin \alpha \cos \beta
\end{array}\right]
$$

and we can write:

$$
\gamma=-\arcsin \left(\left(-s_{\theta} c_{\alpha} c_{\beta}+s_{\phi} c_{\theta} s_{\beta}+c_{\phi} c_{\theta} c_{\beta} s_{\alpha}\right)\right)
$$

Observe that when $\phi=0$ and $\beta=0$, we get the classical formula $\gamma=\theta-\alpha$.

Here we suppose that during a portion of its flight, the orthogonal projection of the aircraft position over a local horizontal plane follows a horizontal track, given by the coordinates of its points according to a curvilinear abscissa:

$$
x(s), y(s) \quad 0 \leq s \leq s_{f}
$$

where $s=0$ and $s=s_{f}$ correspond respectively to the initial and the final points of the horizontal track. Let $d \vec{s}$ be an elementary portion of the horizontal track starting at point $(x(s), y(s))$, we have:

$$
d \vec{s}=(d x, d y)^{\prime} \text { and }\|d \vec{s}\|=\sqrt{d x^{2}+d y^{2}}=|d x| \sqrt{1+(d y / d x)^{2}}
$$

where $\psi=\arctan (d y / d x)$ is the heading of the tangent to the horizontal track at point $(x(s), y(s))$. Here we will assume that the considered portion of the horizontal track is such as the variation of $d x$ and the variation $d s$ of the curvilinear abscissa have the same sign. Then $d s=d x \sqrt{1+(\operatorname{tg} \psi)^{2}}$ where $d s(d s>0)$ is the distance travelled along the track during a time period $d t$. Then at the limit $(d t \rightarrow 0)$, we get $\dot{s}=\dot{x} \sqrt{1+(\operatorname{tg} \psi)^{2}}$ where $\psi=\arctan (\dot{y} / \dot{x})$ is the heading of the tangent to the horizontal track at point $(x, y)$. When considering the evolution of the aircraft over this horizontal track we can write:

$$
\begin{gathered}
\|\dot{\vec{s}}\|=\sqrt{\dot{x}^{2}+\dot{y}^{2}}=\sqrt{(V \cos \gamma \cos \psi)^{2}+(V \cos \gamma \sin \psi)^{2}} \\
\text { or }\|\dot{\vec{s}}\|=V \cos \gamma
\end{gathered}
$$

\section{AIRCRAFt FLIGHT GUIDANCE DyNAMICS}

Here we introduce the flight guidance dynamics of a transportation aircraft. These equations are extracted from global flight dynamics by deleting the fast rotational dynamics composed of the Euler equations relating attitude angles rates $(\dot{\theta}, \dot{\phi}, \dot{\psi})$ and the components of rotational speed $(p, q, r)$ and the moment equations providing rotational speed rates $(\dot{p}, \dot{q}, \dot{r})$. The main entries for the guidance dynamics are the path angle $\gamma$, the bank angle $\phi$ and the thrust $T$. The flight guidance dynamics are first introduced with respect to time as the independent parameter, then they are rewritten once the curvilinear abscissa is taken as the independent parameter.

\section{A. Classical Aircraft Flight Guidance Dynamics}

Let $x(t), y(t), z(t)$ represent the coordinates of the center of gravity of an aircraft at time $t$. Let $V(t)$ be the modulus of the speed of the aircraft at time $t$, let $\gamma$ be the path angle and let $\psi$ be the heading angle. Then the rate of change with respect to time of the aircraft position coordinates in a local Earth frame is given by:

$$
\dot{x}=V \cos \gamma \cos \psi \quad \dot{y}=V \cos \gamma \sin \psi \quad \dot{z}=-V \sin \gamma
$$

The longitudinal acceleration equation can be approximated by:

$$
\dot{V}=\frac{T(z, V, Q)-D(z, V)}{m}-g \sin \gamma
$$

where $T(z, V, Q)$ and $D(z, V)$ are respectively the engine thrust and the drag force. Here $m$ is the mass of the aircraft and writing $Q$ the fuel flow $(\mathrm{kg} / \mathrm{s})$, then:

$$
\dot{m}=-Q
$$

The heading rate is given by:

$$
\dot{\psi}=\frac{L(z, V)}{m V} \frac{\sin \phi}{\cos \gamma}
$$

The path angle rate is given by:

$$
\dot{\gamma}=\frac{L(z, V) \cos \phi}{m V}-\frac{g \cos \gamma}{V}
$$

The engine dynamics are approximated by a first order system with a time constant $\tau$ and where $T$ is the developed thrust while $T_{c}$ is the thrust setting by the auto-throttle computer:

$$
\dot{T}=\left(T_{c}-T\right) / \tau
$$

\section{B. Space Referenced Aircraft Flight Dynamics}

Here we adopt the following notation:

$$
\frac{d^{k} u}{d s^{k}}=u^{[k]}
$$

where $u$ is any physical variable depending of the curvilinear abscissa $s$. Then taking into account relation (7), we can write the aircraft flight dynamics with respect to space as:

$$
x^{[1]}=\cos \psi \quad y^{[1]}=\sin \psi \quad z^{[1]}=-\operatorname{tg} \gamma
$$


The longitudinal acceleration equation is now given by:

$$
V^{[1]}=\frac{T-D}{m V \cos \gamma}-\frac{g}{V} \operatorname{tg} \gamma
$$

The mass equation is given now by:

$$
m^{[1]}=-\frac{Q}{V \cos \gamma}
$$

The heading angle is such as:

$$
\psi^{[1]}=\frac{L(z, V)}{m V^{2}} \frac{\sin \phi}{(\cos \gamma)^{2}}
$$

The dynamics of the bank angle $\phi$ being neglected for the other inputs to the guidance dynamics we have now:

$$
\gamma^{[1]}=\frac{L(z, V)}{m V^{2}} \frac{\cos \phi}{\cos \gamma}-\frac{g}{V^{2}}
$$

and

$$
T^{[1]}=\left(T_{c}-T\right) /(\tau V \cos \gamma)
$$

Now overfly time obeys to the equation:

$$
t^{[1]}=\frac{1}{V \cos \gamma}
$$

Here the evolution of $x(s)$ and $y(s)$ are given as well as the heading angle $\psi$ by the reference horizontal track. Equation 8.4 provides the necessary value of the bank angle $\phi$ at position $s$ when the aircraft flying at level $z$ with speed $V$ presents a path angle $\gamma$ and a heading rate $\dot{\psi}$. A nonlinear state representation taking $(z, V, m, t)$ as state vector and $\gamma$ and $T$ as inputs can be considered for control. However, the overfly time $t(s)$ of position $s$ is in fact an output variable since there is no interacts with other state variables.

\section{2D+T TRAJECTORY OPTIMIZATION}

Adopting the spatial referenced representation of aircraft guidance dynamics it is possible to formulate more easily vertical trajectory optimization problems involving explicit time constraints. For example, if the problem is to find an energy optimal vertical trajectory along a given horizontal track towards a landing point with different overfly time constraints and a final time constraint, this problem can be written as:

$$
\min _{z, V, Q, \gamma} \int_{s=s_{0}}^{s=s_{f}} Q(s) \cdot d s
$$

with overfly time conditions:

$$
t\left(s_{i}\right)=t_{i} \quad i=1, \cdots, N
$$

with the guidance state equations:

$$
\begin{gathered}
z^{[1]}=-\operatorname{tg} \gamma \\
V^{[1]}=\frac{T(z, V, Q)-D(z, V)}{m V \cos \gamma}-\frac{g}{V} \operatorname{tg} \gamma \\
m^{[1]}=-\frac{Q}{V \cos \gamma} \\
t^{[1]}=\frac{1}{V \cos \gamma}
\end{gathered}
$$

with the lower and upper bound constraints:

$$
\begin{gathered}
z_{\text {min }}(s) \leq z(s) \leq z_{\text {max }}(s) \\
V_{\text {min }}(s) \leq V(s) \leq V_{\text {max }}(s) \\
Q_{\text {min }} \leq Q(s) \leq Q_{\max } \\
\gamma_{\min }(s) \leq \gamma(s) \leq \gamma_{\max }(s)
\end{gathered}
$$

with initial conditions at $s=s_{0}$ :

$$
z\left(s_{0}\right)=z_{0} \quad V\left(s_{0}\right)=V_{0} \quad m\left(s_{0}\right)=m_{0} \quad t\left(s_{0}\right)=0
$$

and final conditions at $s=s_{f}$ :

$$
z\left(s_{f}\right)=z_{f} \quad V\left(s_{f}\right)=V_{f} \quad t\left(s_{f}\right)=t_{f}
$$

The altitude bound constraints limit the feasible vertical space. They are such that at initial position $\left(s=\mathrm{s}_{0}\right)$ :

$$
z_{\text {min }}\left(s_{0}\right)=z_{0}=z_{\text {max }}\left(s_{0}\right)
$$

and at final position $\left(s=s_{f}\right)$ :

$$
z_{\text {min }}\left(s_{f}\right)=z_{f}=z_{\text {max }}\left(s_{f}\right)
$$

Speed bound constraints are such as at $s=s_{0}$ :

and at $s=s_{f}$

$$
V_{\min }(0)=V_{0}=V_{\text {max }}(0)
$$

$$
V_{\text {min }}\left(s_{f}\right)=V_{f}=V_{\text {max }}\left(s_{f}\right)
$$

Here $Q_{\min }$ and $Q_{\max }$ are the minimum and maximum allowed fuel flow rates.

A solution of this problem using optimality conditions from optimal control theory (Minimum Principle) appears improbable considering that this optimal control problem is over constrained. However, since the initial state is totally specified, it appears that Direct Dynamic Programming applied to a spatial discretization of this problem can 
provide straightforwardly a numerical solution [4], [5]. The optimal trajectory can then be summarized by:

$$
z^{*}(s), V^{*}(s) \quad s \in\left[s_{0}, s_{f}\right]
$$

\section{Vertical Flight Control with Spatial Reference}

In this section we consider the design of a control law to follow accurately a spatial referenced optimal trajectory solution of the optimal problem considered at the previous section with for example only a final time condition. We study the case of an approaching aircraft for landing and consider that the final conditions correspond to the flare initialization maneuver.

\section{A. Aircraft Pitch Dynamics}

The motion of an approach/descent transportation aircraft along a landing trajectory are here referenced with respect to a RRF(Runway Reference Frame) where its origin is located at the runway entrance as shown in Figure 1.

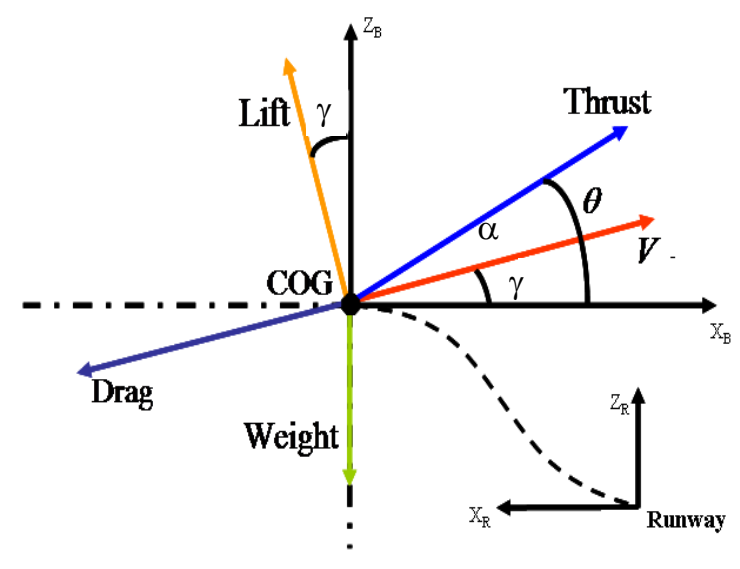

Fig.1 Vertical Flight parameters and forces

Here we consider that the horizontal track to be followed towards the runway is a straight line, so we have: $\phi=0, \psi=c s t$. For this control problem, the pitch dynamics as well as the thrust dynamics (equation 10.6) are to be considered. Then, introducing the ground speed $V_{G}=V \cos \gamma$, the pitch dynamics are given by:

$$
q^{[1]}=\frac{\dot{q}}{V_{G}}=\frac{M}{I_{y} V_{G}}
$$

and

$$
\theta^{[1]}=q / V_{G}
$$

where $I_{y}$ is the inertia moment according to the aircraft lateral axis and $M$ is the pitch moment:

$$
M=\frac{1}{2} \rho V^{2} S \bar{c}\left(C_{m_{0}}+C_{m_{\alpha}}(\theta-\gamma)+C_{m_{q}} \frac{q \bar{c}}{2 V}+C_{m_{\delta_{e}}} \delta_{e}\right)
$$

where $\delta_{e}$ represents the elevator deflection, $\rho$ is the volume mass and $\bar{c}, C_{m_{0}}, C_{m_{\alpha}}, C_{m_{q}}, C_{m_{\delta_{e}}}$ are aerodynamic parameters characteristic of pitch dynamics.

\section{B. Vertical Trajectory control}

Here the considered control objectives are:

- To follow accurately a space-referenced vertical profile $z^{*}(x)$ in accordance with economic and environmental constraints,

- To respect a desired time table $t^{*}(x)$ for its progress towards the runway in accordance with air traffic management considerations.

This second objective can be replaced by following the corresponding speed profile $V^{*}(x) \quad x \in\left[x_{0}, x_{f}\right]$.

It is possible to write:

$$
\begin{gathered}
z^{[3]}=\frac{1}{V_{G}^{2}}\left[\begin{array}{c}
A_{z}(z, \theta-\gamma, V, T) \\
+B_{z_{q}}(z, \theta-\gamma, V, T) q \\
+B_{z_{T}}(z, \theta-\gamma, V, T) T_{C}
\end{array}\right] \\
V_{\text {air }}^{[2]}=\frac{1}{V_{G}^{2}}\left[\begin{array}{c}
A_{V}(z, \theta-\gamma, V, T) \\
+B_{V_{q}}(z, \theta-\gamma, V, T) q \\
+B_{V_{T}}(z, \theta-\gamma, V, T) T_{C}
\end{array}\right]
\end{gathered}
$$

where the rather complex expressions of components $A_{V}$, $B_{V_{q}}, B_{V_{T}}$ and $A_{z}, B_{z_{q}}, B_{z_{T}}$ are detailed in [6]. Then, Non Linear Inverse control techniques [7], [8] and [9], can be applied since outputs $z$ and $V$ present relatives degrees 3 and 2 with respect to control $q$ and $T_{c}$. This induces the absence of internal dynamics while since in general flight conditions the control matrix given by:

$$
\left(\begin{array}{ll}
B_{z_{q}} & B_{z_{T}} \\
B_{V_{q}} & B_{V_{T}}
\end{array}\right)
$$

is invertible, it is possible to invert the nonlinear flight dynamics to get online the corresponding inputs. Introducing the altitude and speed tracking errors as:

$$
\begin{aligned}
& \xi_{z}(x)=z(x)-z^{*}(x) \\
& \xi_{V_{a i r}}(x)=V(x)-V^{*}(x)
\end{aligned}
$$

As shown in [6], we can now compute control laws which impose to this tracking errors linear decoupled dynamics such as:

$$
\begin{aligned}
& \sum_{k=0}^{3} a_{k}^{z} \xi_{z}(x)^{[k]}=0 \\
& \sum_{k=0}^{2} a_{k}^{V} \xi_{V}(x)^{[k]}=0
\end{aligned}
$$


where the corresponding characteristic polynomials are chosen to be asymptotically stable with adequate transients and response times.

So we get:

$$
\left(\begin{array}{c}
q \\
T_{C}
\end{array}\right)=\left(\begin{array}{ll}
B_{z_{q}} & B_{z_{T}} \\
B_{V_{q}} & B_{V_{T}}
\end{array}\right)^{-1} \times\left(\begin{array}{c}
V_{G}^{2} D_{z}(x)-A_{z} \\
V_{G}^{2} D_{V_{\text {air }}}(x)-A_{V}
\end{array}\right)
$$

with:

$$
\begin{gathered}
D_{z}(x)=z_{d}^{*[3]}(x)+k_{1 z} \xi_{z}^{[2]}(x)+k_{2 z} \xi_{z}^{[1]}(x)+k_{3 z} \xi_{z}(x) \\
D_{V_{\text {air }}}(x)=V^{*[2]}(x)+k_{1 v} \xi_{V_{\text {air }}}^{[1]}(x)+k_{2 v} \xi_{V_{\text {air }}}(x)
\end{gathered}
$$

Observe here that while the spatial derivatives of desired outputs $z^{*}(x)$ and $V^{*}(x)$ can be directly computed, the successive spatial derivatives of actual outputs $z(x)$ and $V(x)$ can be computed by spatial derivation of the corresponding state equations.

\section{Simulation Study}

The proposed spatial control approach is illustrated using the Research Civil Aircraft Model (RCAM) which has the characteristics of a wide body transportation aircraft [10] with a maximum allowable landing mass of about 125 tons with a nominal landing speed of $68 \mathrm{~m} / \mathrm{s}$.

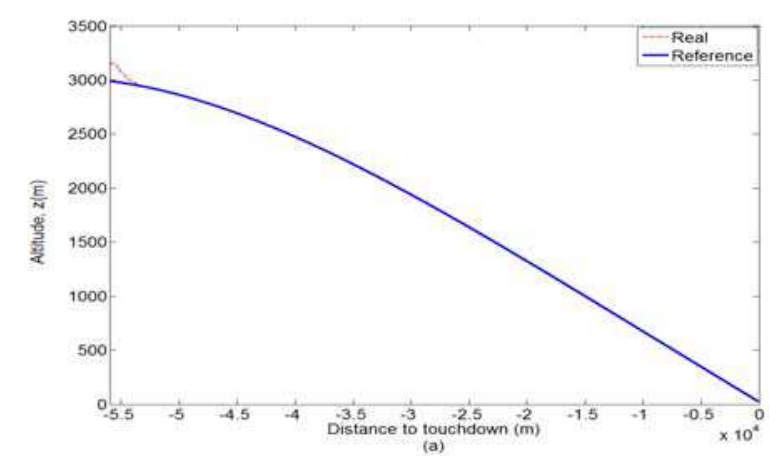

Fig. 2 Trajectory Tracking Performance by Space Control

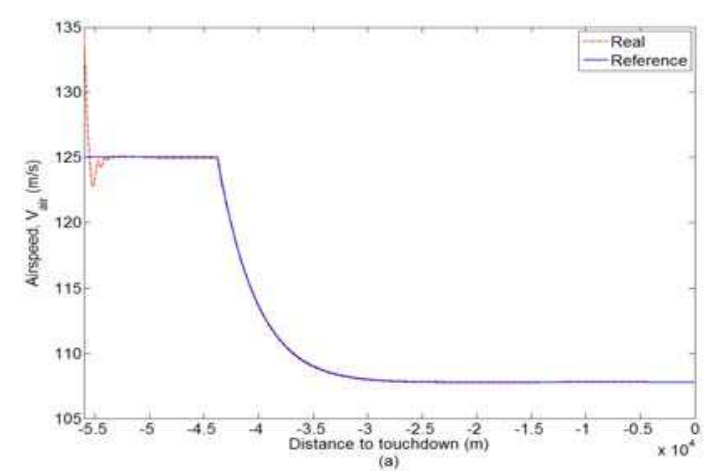

Fig 3 Speed Tracking Performance by Space Control
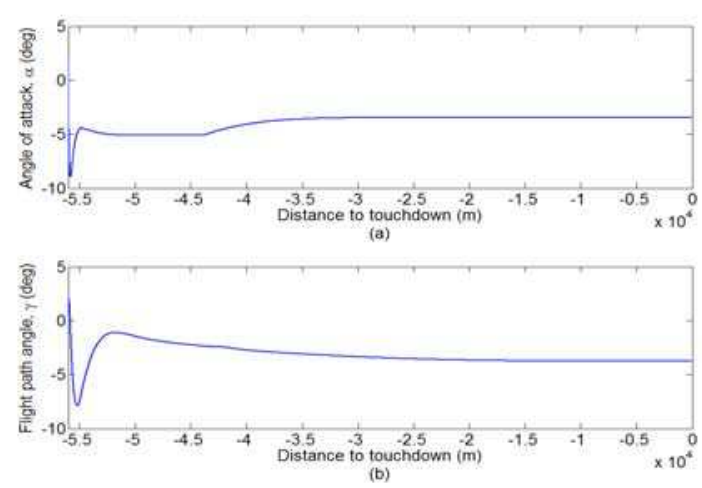

Fig 4 Angle of Attack $(\theta-\gamma)$ and Flight Path Angle $\gamma$ Evolutions
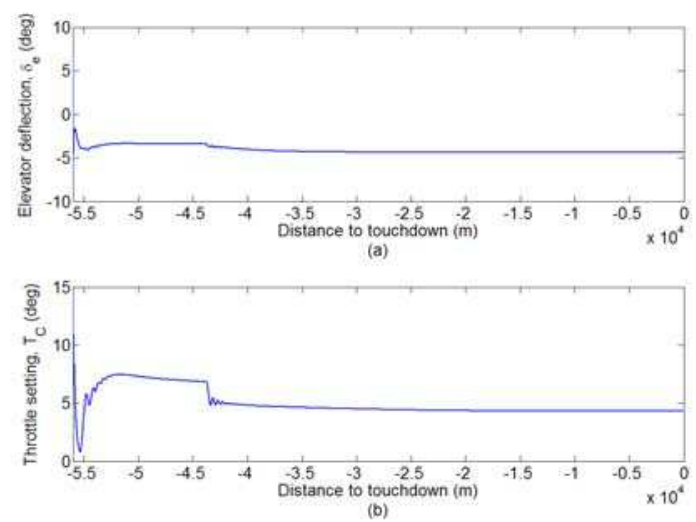

Fig. 5 Control Inputs Evolution with Space Control

From the above simulation results it appears that the proposed approach is feasible and provide accurate trajectory tracking performance.

\section{CONCLUSION}

In this communication we have considered the use of a spatial reference to provide new capabilities for aircraft operating within a more and more dense traffic. One of the objectives has been to cope more efficiently with overfly and arrival time constraints. The trajectory optimization problem corresponding to reference trajectory generation at the level of flight management, as well as time constrained vertical trajectory tracking at the level of flight guidance have been considered. A new formulation for the trajectory optimization problem, including explicit time constraints, has been proposed. Then a new longitudinal guidance scheme for transportation aircraft has been proposed. The main objective here has been to improve the tracking accuracy performance of the guidance along the optimal trajectory generated within the spatial reference frame. This has led to the development of a new representation of longitudinal flight dynamics where the independent variable is ground distance to a reference point. The nonlinear inverse control technique has been applied in this context so 
that tracking errors follow independent and asymptotically stable spatial dynamics around the desired trajectories. It appears that the proposed approach results in good performances as well as in an enhanced track predictability.

To get applicability this new approach still should overcome important challenges related mainly with navigation and online wind estimation performances. Then an improved integration of on board flight path optimization functions including the consideration of neighbouring traffic and the guidance function, will become possible.

\section{REFERENCES}

[1] Bernd Korn, Alexander Kuenz, 2006, 4D FMS for Increasing Efficiency of TMA Operations, USA, IEEE/AIAA 25th Digital Avionics Systems Conference, pp. 1E4-1-1E4-8.

[2] Shih-Yih Young, Real-Time 4-D Trajectory Planning for RNP Flight Operations, IEEE Integrated communications, Navigation and Surveillance Conference, pp. 1-9, 2009.

[3] Etkin B, 1985, Dynamics of Atmospheric Flight, New York, John Wiley and Sons, Inc.

[4] Hagelauer P. and F. Mora-Camino 1998, A Soft Dynamic Programming Approach for On-line Aircraft 4D Optimization, European Journal of Operations Research, Vol.107, pp. 87-95.

[5] Wu H., N. Chio Cho, H. Bouadi, L. Zhong and F. Mora-Camino, 2012, Dynammic Programming for Trajectory Optimization of Engine-Out Transportation Aircraft, Chinese Control and Decision Conference-CCDC, pp. 98-103.

[6] Bouadi. H and F. Mora-Camino, August 13-16, 2012, Aircraft Trajectory Tracking by Nonlinear Spatial Inversion, Minneapolis, Minnesota, USA, AIAA Guidance, Navigation and Control Conference.

[7] Singh S.N. and W.J. Rugh, 1972, Decoupling in a Class of Nonlinear Systems by State Feedback, ASME Journal of Dynamic Systems, Measurement, and Control, Series G, Vol. 94, pp. 323-329.

[8] I.J.-J. Slotine and L. Weiping, 1991, Applied Nonlinear Control, Prentice Hall, Upper Saddle River, NJ.

[9] A. Drouin, O. Lengerke, A.B. Ramos and F. Mora-Camino,2012, Rotorcraft Trajectory tracking by Supervised NLI control, Proceedings of International Multiconference of Engineers and Computer Scientist, vol II.

[10] Magni J-F. et al, Robust Flight Control, A Design Challenge, Springer-Verlag, London. 